Meta

Journal des traducteurs

Translators' Journal

\title{
Les traducteurs camerounais face aux problèmes linguistiques
}

\section{Thérèse Priso}

Volume 28, numéro 1, mars 1983

La traduction dans le monde

Translation around the World

URI : https://id.erudit.org/iderudit/003870ar

DOI : https://doi.org/10.7202/003870ar

Aller au sommaire du numéro

Éditeur(s)

Les Presses de l'Université de Montréal

ISSN

0026-0452 (imprimé)

1492-1421 (numérique)

Découvrir la revue

Citer cet article

Priso, T. (1983). Les traducteurs camerounais face aux problèmes linguistiques. Meta, 28(1), 20-26. https://doi.org/10.7202/003870ar d'utilisation que vous pouvez consulter en ligne.

https://apropos.erudit.org/fr/usagers/politique-dutilisation/ 


\section{LES TRADUCTEURS CAMEROUNAIS FACE AUX PROBLÈMES LINGUISTIQUES}

THÉRÈSE PrISO

Rappelons brièvement l'expérience camerounaise en matière de traduction, en ce qui concerne notamment la nature des textes à traduire et les problèmes spécifiques de traduction qui se posent en milieu camerounais.

Il sera ensuite possible, en connaissance du cadre social et politique, de se pencher sur les illustrations linguistiques.

\section{L'EXPÉRIENCE CAMEROUNAISE EN TRADUCTION}

La prise de conscience de l'importance de la traduction au Cameroun a évolué en raison des problèmes posés par une administration désormais bilingue au lendemain de la Réunification des deux Camerouns : le Cameroun occidental anglophone où l'on parle notamment l'anglais et le Pidgin English et le Cameroun oriental francophone où l'on parle notamment le français et un peu de Pidgin English dans certaines régions comme le littoral, en particulier dans la ville de Douala, capitale économique du pays. Il importe de signaler que sur toute l'étendue de la Jeune Fédération ainsi née le $1^{\text {er }}$ octobre 1961, plus de 200 langues maternelles et autres dialectes étaient couramment parlés!

Face à cette situation linguistique particulière qui avait conduit à l'adoption de deux langues officielles, l'anglais et le français, la traduction s'avère un important facteur de communication, un pont entre les deux groupes linguistiques majoritaires. Les traducteurs et les interprètes sont considérés comme les intermédiaires indispensables tout indiqués pour assurer le dialogue et la compréhension nécessaires entre les anglophones et les francophones.

Ainsi donc, la traduction au Cameroun est née de la nécessité impérieuse de communiquer entre les citoyens de culture et de langue d'instruction anglaises et ceux de culture et de langue d'instruction française ${ }^{1}$.

\section{NATURE DES TEXTES TRADUITS}

Le double caractère politique et linguistique de la traduction au Cameroun a eu une grande influence sur la nature des textes à traduire, lesquels sont essentiellement officiels. En outre, le plus gros volume de travail se fait vers l'anglais, à la différence du Canada, les Camerounais d'expression anglaise étant minori-

1. Chaque camerounais parle d'abord sa langue maternelle ou un dialecte quelconque avant d'apprendre l'anglais ou le français à l'école. 
taires. La répartition du travail est donc inégale entre les traducteurs de la Section de langue anglaise et ceux de la Section de langue française. Il peut ainsi arriver que les francophones soient plus ou moins «au chômage» pendant que leurs collègues anglophones sont submergés de travail, ce qui rend encore plus difficile l'évaluation du rendement individuel des traducteurs. Mais il semble que ce problème d'évaluation de la quantité et de la qualité des prestations des traducteurs soit commun à tous les services et organes de traduction...

a) Parmi les documents officiels soumis à la traduction, il y a d'abord les Discours du Président de la République. À titre d'exemple, on peut citer les discours d'ouverture et de cloture des sessions de l'Assemblée nationale, les réponses aux présentations des Lettres de créance des nouveaux ambassadeurs, les messages à la nation, les rapports de politique générale à l'occasion des congrès de l'Union Nationale Camerounaise (UNC), notre grand Parti de masse. Ces congrès se tiennent tous les cinq ans et leurs actes sont intégralement traduits dans les deux langues officielles.

b) Le Journal officiel (Official Gazette) se taille la part du lion dans le volume de travail imparti exclusivement aux traducteurs de langue anglaise ${ }^{2}$. II comporte notamment des décrets, des lois, des ordonnances, des arrêtés, des textes domaniaux divers. C'est un bimensuel bilingue qui publie de temps en temps les Statuts de certains corps de fonctionnaires camerounais. Il présente certains points communs avec HANSARD et surtout avec la Gazette Provinciale du Québec.

c) Viennent ensuite les lettres officielles émanant des Affaires étrangères et d'autres ministères, des ambassades et d'autres pays (par exemple, les offres de bourses des gouvernements étrangers).

d) Les rapports des gouverneurs, des préfets, des sous-préfets et des chefs de district, ainsi que les rapports d'activité et de gestion de certaines sociétés, occupent une bonne place dans cette traduction officielle. Il en est de même des travaux réalisés par des spécialistes. Je pense par exemple aux rapports du Dr LANTUM après ses enquêtes sur la fécondité, sur la nutrition, sur la planification sanitaire régionale...

e) Les programmes officiels d'enseignement et tous les diplômes et attestations diverses libellés en d'autres langues que le français sont préalablement traduits et revêtus du cachet officiel attestant de leur authenticité avant d'être envoyés au Service des équivalences du ministère de l'Éducation nationale.

f) Les articles de presse d'intérêt national parus dans les grands quotidiens étrangers.

g) Les slogans et les programmes des fêtes et des manifestations officielles.

h) La correspondance individuelle des citoyens (surtout de langue anglaise) adressée au Chef de l'État ou au Secrétaire général de la Présidence pour solliciter de l'aide (financière ou morale) et des emplois, pour revendiquer certains droits professionnels, ou pour exposer des problèmes très personnels...

2. Les autres traductions réservés aux seuls anglophones sont le Plan quinquenal et le Budget annuel, préalablement rédigés en français. 
Telle est la nature des textes traduits au service de traduction officiel de la Présidence de la République dénommé Direction des services linguistiques depuis août 1980. Avant cette date, il avait successivement porté les noms de Bureau linguistique et de Service linguistique.

i) Parallèlement à la Direction des services linguistiques, fonctionne le Service de traduction de l'Assemblée nationale. Il comprend un corps de traducteurs et d'interprètes ayant reçu une formation professionnelle complète comme ceux de la Présidence de la République, mais accomplissant des tâches différentes. En effet, les traducteurs de l'Assemblée nationale assurent la traduction des textes parlementaires et celle des écrits et documents du Parti, par exemple les articles publiés dans L'UNITÉ, journal du Parti.

Avant de clore le présent chapitre, il convient de dire un mot sur les destinataires de ces traductions essentiellement officielles. Comme l'on s'en doute, elles intéressent au premier chef la Présidence de la République, l'Assemblée nationale, les divers ministères et les diverses institutions publiques et parapubliques. Seuls les slogans et les programmes des fêtes sont à la portée du public camerounais tout entier.

\section{PROBLÈMES SPÉCIFIQUES DE TRADUCTION}

Les problèmes spécifiques auxquels les traducteurs et les traducteursinterprètes camerounais ont à faire face peuvent être classés dans trois grandes catégories selon l'origine proche ou lointaine de leur difficulté. Nous traiterons donc successivement: des problèmes sémantiques, des problèmes syntaxiques, des problèmes socio-culturels.

\section{a) Problèmes sémantiques}

i) Ceux-ci résultent en grande partie d'une façon maladroite d'exprimer des idées autrement originales et intéressantes. Je me rappelle par exemple une phrase d'une requête que j'avais eue à traduire il y a quelques années. L'auteur de la lettre se plaignait d'avoir été abusé par un fonctionnaire qui lui avait promis un emploi bien que, disait-il : «I gave him a goat and a bottle of a white horse!»

Il me fallut évidemment faire le lien entre «a white horse» et la marque de whisky «White Horse»... Et je traduisis la phrase par: «Je lui ai offert une chèvre et une bouteille de whisky (marque White Horse)!»

ii) Un autre problème d'ordre sémantique provient du fait que, pour les camerounais anglophones, certains mots ne recouvrent pas tout à fait la même aire sémantique que pour les citoyens francophones. C'est cette situation qui a motivé, à l'origine, la publication des Notes de traduction, puis des Notes de terminologie, lesquelles sont présentées indifféremment en anglais ou en français.

Comme leur nom le suggère, ces Notes guident le traducteur camerounais à travers les pièges du vocabulaire employé dans notre contexte en même temps qu'elles visent à harmoniser ce vocabulaire, à uniformiser cette terminologie afin que les anglophones et les francophones «disent véritablement la même chose" , qu'ils se réfèrent à la même situation lorsqu'ils s'expriment chacun dans sa langue. À cet égard, il convient de noter l'importance de la Terminology Note 
no. 83 of 5 march 1979: Names of Administrative Units and of Persons in Charge of Their Administration.

Enfin, les Notes de terminologie allègent beaucoup la tâche du réviseur qui n'a plus à s'arrêter sur la signification même des énoncés linguistiques, mais à s'occuper davantage à remodeler, à restructurer, bref, à "polir» les textescibles.

C'est à ce niveau qu'apparaît l'importance capitale des notions de «situation», d' «équivalences de situations», d' «équivalences contextuelles» etc., et de leur incidence sur la traduction. En effet, en raison des difficultés d'ordre sémantique qui résultent le plus souvent d'ailleurs des écarts culturels entre nos deux groupes linguistiques, seule la référence à «la même situation » permet au traducteur camerounais de trouver en langue d'arrivée «l'équivalent naturel le plus proche» de la langue de départ.

Il existe de nombreux exemples illustrant l'emploi abusif, par les anglophones, des termes «officer», "clerk» et «chief» employés avec d'autres mots et dont la traduction varie selon les contextes. Citons, entre autres :

- Appeals officer $=$ employé du service du contentieux

- Assistant Subdivisional officer $=$ adjoint d'arrondissement

- Community Development officer $=$ agent du développement communautaire

- Chief Collector = receveur en chef

- Chief Clerk = commis principal, chef de bureau

- Chief Secretary = secrétaire général

- Dispatch Clerk = commis aux expéditions

Parallèlement à ces mots qui viennent très souvent dans des textes, il existe d'autres termes et expressions à la traduction figée mais qui, comme on va le voir, ne va pas de soi pour un traducteur étranger: les équivalences s'établissent nécessairement en s'aidant de la situation décrite implicitement dans le texte-source. Voici quelques exemples assez éloquents :

- Tax drive $=$ campagne de recouvrement des impôts

- Identification campaign = contrôle, vérification des pièces d'identité

- Meet-the-people-tour $=$ tournée de prise de contact (par un responsable des populations de son aire de juridiction, de ses «administrés».

- On official tour $=$ en visite officielle

- Native Authority/Authorities = administration locale (par les autochtones cux-mêmes)

- House of Chiefs = assemblée des chefs traditionnels

- Honorable Members = parlementaires, députés.

- Chieftancy dispute = querelle au sujet de l'élection/de la désignation d'un chef traditionnel; conflit de chefferies

- Apex Organization = fédération des coopératives

- Urban Council = commune de plein exercice $(\mathrm{CPE})$

- Area Council $=$ commune mixte rurale $(\mathrm{CMR})$

- Handing Over Notes = rapport de passation de service

- Dirt Road = Piste (et par extension, route non asphaltée).

- Evacuation roads $=$ pistes de collecte (des vivres à destination des marchés locaux éloignés).

Voici maintenant quelques «expressions casse-tête» pour les traducteurs de langue anglaise :

- Écoles sous l'arbre

- Case de passage.

iii) Les traducteurs camerounais, on s'en doute, connaissent l'éternel problème d'interférence linguistique qui se pose dans toutes les sociétés où deux 
langues au moins sont en contact permanent. Je crains, en ce qui concerne le Cameroun, que ce problème s'aggrave au fur et à mesure que le bilinguisme deviendra une réalité vraiment quotidienne, au fur et à mesure qu'un plus grand nombre de Camerounais s'exprimeront couramment en anglais et en français, dans les circonstances les plus ordinaires de la vie quotidienne.

Déjà à l'heure actuelle où seule une minorité, l'élite en quelque sorte, parle les deux langues officielles nationales, le phénomène d'interférence linguistique gagne insidieusement du terrain.

On pourrait déjà appuyer sur la sonnette d'alarme lorsque l'on rencontre dans un texte ou que l'on entend dans des conversations des tournures comme celles-ci :

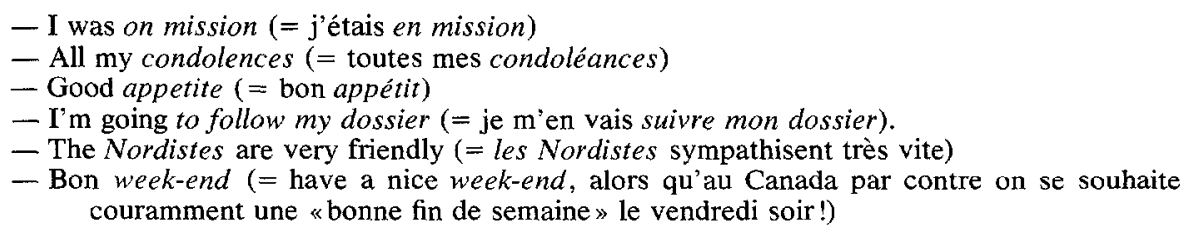

Un fait significatif mérite d'être signalé ici. C'est qu'au Cameroun, les anglophones étant minoritaires, ils subissent davantage les effets du phénomène d'interférence linguistique, d'autant plus qu'ils sont toujours enclins, quand ils se retrouvent plusieurs, à parler le Pidgin English plutôt que l'Anglais.

Tout le problème est de savoir de quelle manière on peut rétablir l'équilibre linguistique ainsi rompu entre le groupe dit «minoritaire» et celui reconnu comme étant «majoritaire».

\section{b) Problèmes syntaxiques}

i) Ils relèvent notamment de la mauvaise rédaction des textes originaux dans lesquels l'orthographe et la grammaire laissent souvent à désirer. Il s'ensuit que le style est confus. D'une manière générale, il s'agit de textes rédigés dans un mauvais anglais ou dans un français à peine lisible et difficile à déchiffrer.

ii) Un autre problème crucial est celui du non-respect des niveaux de langue. En effet, les anglophones sont directs, sans protocole. Ils détestent les intermédiaires dont ils se méfient: ils préfêrent «avoir affaire au Bon Dieu plutôt qu'à ses saints»! Ils s'adressent donc directement aux responsables, au Chef de l'État lui-même, dans un langage simple et sans artifice qui rend la compréhension difficile au traducteur de langue française. Celui-ci est souvent tenu d'élaborer le texte, de le rendre dans un style administratif convenable et acceptable. Dans notre contexte en effet, on ne s'adresse pas au Président de la République comme à un ministre ou à un collègue de bureau, et un bon planteur de cacao de Monatele ne s'exprime pas comme un administrateur civil!

Ainsi donc, la traduction sauve souvent de nombreuses requêtes qui auraient été froissées et jetées dans le panier à ordures et leurs auteurs peut-être poursuivis pour avoir ... mal exprimé des idées valables. 


\section{c) Problèmes socio-culturels}

i) Les problèmes socio-culturels rencontrés en traduction proviennent des différences socio-linguistiques et culturelles entre les Camerounais anglophones et les Camerounais francophones. La situation est d'autant plus préoccupante que malgré l'adoption de deux langues officielles en 1961, lors de la Réunification des deux Camerouns, les langues maternelles vernaculaires continuent d'occuper le devant de la scène linguistique et de rivaliser d'influence entre elles. Très souvent, leurs concepts, leurs expressions idiomatiques sont transférés et traduits littéralement en anglais ou en français, selon le cas. Quand il s'agit de mots ou notions intraduisibles, le traducteur doit les conserver tels quels dans le texte-cible, évitant ainsi d'embrouiller les lecteurs avec de longues définitions ou des explications au bas de la page.

Voici, à titre indicatif, quelques-uns de ces emprunts à des langues locales (Provinces du Nord, du Nord-Ouest et du Sud-Ouest en particulier) :

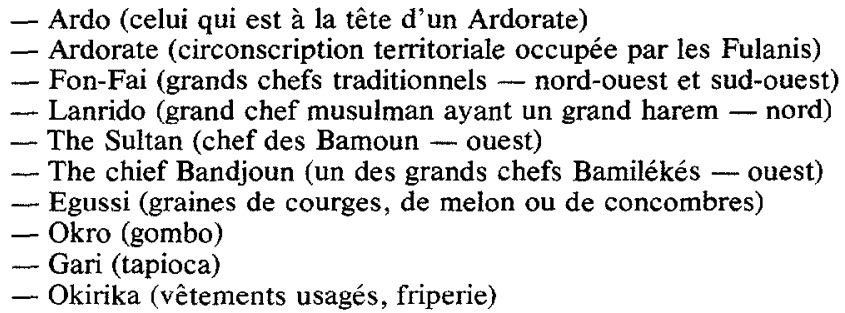

Parfois, le traducteur de langue française est obligé d'adopter en français la traduction d'un mot passé tel quel en anglais, c'est-à-dire emprunté aux langues vernaculaires. C'est par exemple le cas de «Jangali tax», que nous rendons par «impôt sur le bétail» après une longue gymnastique intellectuelle! «Jangali» signifie «bétail », «troupeau » dans son dialecte d'origine....

ii) Il importe de noter ici que les problèmes socio-culturels sont indissociables des problèmes sémantiques, dans la majorité des cas. Comme on l'a vu, c'est dans le but de les réduire au minimum, de limiter pour ainsi dire les dégâts dans le cadre de la recherche des équivalences de traduction, que les Notes de traduction et les Notes de terminologie ont été instituées. La Note de terminologie, $\mathrm{n}^{\circ} 76$ du 10 octobre 1978, sur les Remarques générales de traduction, est très significative à cet égard. Les remarques en question (10 pages dactylographiées) portent soit sur la langue et le sens des mots, soit sur des problèmes spécifiques de traduction rencontrés dans des textes.

iii) Les problèmes socio-culturels ont en grande partie inspiré le programme de formation des réviseurs camerounais qui a démarré en juin 1977.

En effet, après avoir longtemps bénéficié de l'expérience des réviseurs expatriés, Anglais et Français, nous nous sommes rendu compte que les nationaux devaient progressivement prendre la relève. Cela devenait d'autant plus pressant que l'expérience avait montré que bien que nos grands maîtres connussent tous les secrets de la langue de Shakespeare et de celle de Molière, ils ignoraient une bonne partie sinon tout de la culture et des réalités camerounaises. De ce fait, ils devaient très souvent faire appel aux lumières des tra- 
ducteurs camerounais pour expliciter tel terme ou expression («tax-drive», "Okirika», "chieftancy dispute»), ou la portée significative d'une phrase ambiguë.

Les réviseurs camerounais devraient être compétents et efficaces pour deux raisons évidentes : $l^{\mathrm{e}}$ ils connaissent bien leur milieu professionnel et tous les problèmes qui s'y posent; $2^{\mathrm{e}}$ ils auront acquis l'expérience de leurs aînés, en traduction comme en révision, dans des organismes internationaux bien connus qui acceptent d'assurer la formation de nos stagiaires. Il s'agit, depuis 1977, de l'UNESCO et de l'OCDE à Paris, de la CEE à Bruxelles et à Luxembourg; du Bureau des traductions au Canada.

\section{CONCLUSION}

Comme on vient de le voir succinctement, l'expérience camerounaise en traduction est essentiellement pratique, je dirais même pragmatique. Née, on s'en souvient, du besoin urgent d'assurer la communication, le dialogue et la compréhension entre les citoyens des deux groupes linguistiques existants, la traduction au Cameroun ne pouvait pas, à l'origine, s'arrêter à l'élaboration d'une formation théorique sous-jacente à son exercice. Il fallait traduire et traduire vite. Et je me permettrai de dire que comme l'on devient forgeron en forgeant, beaucoup de camerounais sont devenus traducteurs en traduisant...

À l'heure actuelle, des recherches n'ont pas encore été entreprises par des Camerounais en vue de la définition et de l'élaboration d'une théorie de base. L'un des plus grands handicaps est le problème d'adaptation, à notre milieu, des méthodes de traduction apprises dans les écoles de formation étrangères. En effet, ces méthodes, comme la nature des textes donnés à titre d'exercices d'entraînement dans les dites écoles, n'ont généralement rien à voir avec le travail pratique fait sur place chez nous. Pour le moment, ce travail ne s'apprend et ne se comprend véritablement que sur le tas. 